\title{
Optimized IDN-FOPD Controller based AGC Loop of a Wind-Solar-Thermal Interconnected Power System with HES and IPFC Units
}

\author{
V. Suresh Babu, K. Shanmukha Sundar
}

\begin{abstract}
The current scenario of this version focuses on automatic generation control (AGC) exploration to a practical power system incorporating a versatile mixture of two-area generating multi-source units under a deregulated format. Each and every area will have two generating and distribution companies termed as Gencos and Discos. In Gencos, area-1 is a combination of wind energy power plant along with a thermal unit while area-2 is a solar power plant and thermal unit. In this research, the thermal plants are well thought-out with reheat tandem compound steam turbine rather than steam turbine dynamic representation constraint is believed to be expected. In this examination, another ID comprises filter with Fractional Order Proportional Derivative (IDN-FOPD) controller is prearranged and carry out for the AGC scheme. The control limits of the IDN-FOPD controller refrained using Lightning Search Algorithm (LSA) and its demonstration is dissimilarity and PI and Fractional Order Proportional Integral Derivative (FOPID) based controllers. Further to improve AGC execution, Hydrogen Energy Storage (HES) is included into its control area and Interline Power Flow Controller (IPFC) is integrated to dealt with Tie line. Simulated results demonstrate that the LSA tuned IDN-FOPD controller progress the vibrant yield reaction of the test system as far as less summit deviation, tie-line power oscillations and settling time of area frequencies in a variety of interactions of the deregulated power system. The implementation of HES and IPFC units successfully detain the initial descend in reappearance just as the tie line divergences in power while an unexpected burden upsetting influence. Subsequently, the coordinated activity of HES-IPFC units in the AGC circle could perk up the system implementation.
\end{abstract}

Keywords: Automatic Generation Control, Hydrogen Energy Storage, IDN-FOPD controller, IPFC, Search Algorithm.

\section{INTRODUCTION}

The power network system is immense scales arrange which contains incalculable generating stations (and generators) interrelated through the network of transmission. In power system, the proportion of generated power is devoted at a comparative minute. Any change of power intends frequency awkwardness. Hence the power systems frequency is a critical execution sign administrator regarding steadiness and safety. Fundamental commitment to framework later an unsettling influence will generally be fulfilled through power plants by their speed representative attributes along with burden recurrence reaction.

Alternatively, discretionary control strategy in a power

Revised Manuscript Received on October 15, 2019.

V. Suresh Babu, Assistant Director, National Power Training Institute /PSTI, Ministry of Power, Bengaluru, Karnataka, India Email: sureshbabu27@gmail.com

K. Shanmukha Sundar, Professor \& Head, Department of EEE, Dayananda Sagar College of Engineering, Bengaluru, Karnataka, India, Email: bonniekhs@gmail.com framework is executed by specific units that are outfitted using a programmed controller that changes the set focuses of speed representative [1]. The basic goal of the automatic generation control is intended to coordinate recurrence at a predefined ostensible worth and keep up the power trade between the controls to oversee recurrence at a foreordained ostensible worth. The consolidated effects of tie line control and the framework recurrence changes are normally considered as the yield of AGC which is termed as Area Control Error (ACE). With reference to the change in ACE in accordance with zero by the automatic generation control, both the errors such as frequency and tie line power will end up towards zero [2]. The present revamped framework comprises of Discos, Gencos, ISO and Transco which are the companies related to distribution, generation, independent system operator and transmission. In open vitality monetary circumstance, independent system operator keeps up the straightforwardness to exchanges satisfying with requests between the generation and distribution companies. The two potential exercises are preceding in the business Poolco oriented exchanges along with reciprocal exchanges [3]. An ISO is a selfadministering expert that deals with the entirety of the trades acknowledged among generation and distribution companies. A Disco Participation Matrix termed as DPM is utilized for pipedream of bonds among Gencos and Discos. An ISO needs to perform assorted right hand organizations for the triumphant movement of the power framework [4].

In view of specific disadvantages of carbon discharge, it is essential to consolidate inexhaustible wellsprings of vitality like hydro, sunlight based and wind vitality. The use of inexhaustible sources decreases the chance of an unnatural weather change. Wind energy conversion system was incorporated onto a network as they are utilized as pinnacle burden plants to protect the steadfast nature of reserve. The recurrence control winds up problematic and consequently testing, inside seeing a breeze farm on account of the spasmodic thought of wind speed. Furthermore, as a result of the cutoff points of regular sources, it is critical to use non-traditional sources to consider the AGC issue [5]. The solar energy is perfect vitality available in overflowing. The change of sunlight based vitality into electric vitality doesn't release ozone-draining substances. Besides, the utilization of this nonconventional vitality lessens the utilization of standard wellsprings of vitality The plausibility of the mix of wind-sun based warm power plant for 
automatic generator control consider, regardless, their examination is inadequate to a disconnected framework just and they have not related any control approach for wind-sun oriented solar thermal power plant [6,7]. Consequently, automatic generator control of multi area system incorporating wind solar thermal combined power plants (WSTPP) is significant for further investigations.

The proximity of imperatives makes a framework further useful that requires profoundly proficient controllers to decrease deviations happening in recurrence, tie line control reactions. The discretionary controller's explicitly integral, proportional integral, proportional integral derivatives (I, PI, PID) are discussed in [8]. As of late, different types of fractional order (FO) in addition to two degree of freedom (2DOF) controllers are elucidated in [7-10]. Falling of controllers can be improved by combining FO and IO controllers. However, falling vital subordinate alongside channel, which is IO and FO corresponding subsidiary controller's utilization isn't evaluated in such arrangement of AGC. Along these lines, here untruths an expansion for extra assessments. A couple of improvement methods expect a significant activity to locate the ideal controller parameters of a couple of standard controllers to deal with the AGC issue. A later extreme calculation based on Meta heuristic termed as Lightning Search Algorithm is an unprecedented along with adaptable progress strategy that was affected by the trademark marvel of lightning [11]. The upsides of this calculation are to be used for the streamlining of PI, FOPID, and IDN-FOPD controller increases of AGC hover for two-territory sunlight based warm interconnected deregulated control framework for various exchanges.

Adjusting power natural market is continually an unusual procedure especially at pinnacle loads. Thusly, there may be not kidding stresses over the dependable activity of the power framework. Along these lines, it is important to incorporate quick Energy Storage Systems having storage capacity along with the kinetic energy of the generator rotors is suggested to soggy out the oscillations of the frequency [12]. Be that as it may, because of the economical reasons, it is unrealistic to place ESS in every one of the areas. Flexible Alternating Current Transmission Systems controllers assume a vital job to control the flow of power in an power system which is interconnected [13]. In this assessment, the possibility of AGC in two-territory windsunlight based warm power framework having composed control activity with Hydrogen Energy Storage (HES) along with Interline Power Flow Controller (IPFC) units are prepared for controlling the system execution in an extraordinarily quick manner and to improve power move restrains so as to have a prevalent rebuilding.

\section{Design of IDN-FOPD controller using Lightning Search Algorithm}

\section{A. Controller structure of IDN-FOPD controller}

The course aggregate of whole number request (IO) and fragmentary request (FO) controller's basic subordinate incorporating channel alongside partial request relative subsidiary (IDN FOPD) is suggested as an assistant controller for the modified AGC framework. The nitty gritty structure of such controller is shown in Fig. 1 in which there are two squares necessary request IDN controller and fragmentary request PD controller. The LSA methodologies [11] are used to choose the perfect prerequisites of IDNFOPD controllers with the target to restrict the Integral square of area control error, which can be defined in the accompanying way:

$$
J=\int_{0}^{t_{\text {sim }}}\left(\beta_{1} \Delta F_{1}^{2}+\beta_{2} \Delta F_{2}^{2}+\Delta P_{\text {tie }}^{2}\right) d t
$$

The formulation of the design problem is shown as below where the controller parameter bounds are the constraints.

$$
\begin{aligned}
& \text { Minimize } \\
& \text { Subject to } \\
& K_{I}^{\text {min }} \leq K_{I} \leq K_{I}^{\text {max }}, K_{D}^{\text {min }} \leq K_{D} \leq K_{D}^{\text {max }}, N^{\text {min }} \leq N \leq \\
& N^{\text {max }}, K_{p}^{\text {min }} \leq K_{p} \leq K_{p}^{\text {max }}, K_{d}^{\text {min }} \leq K_{d} \leq K_{d}^{\text {max }}, \mu^{\text {min }} \leq \\
& \mu \leq \mu^{\text {max }},
\end{aligned}
$$

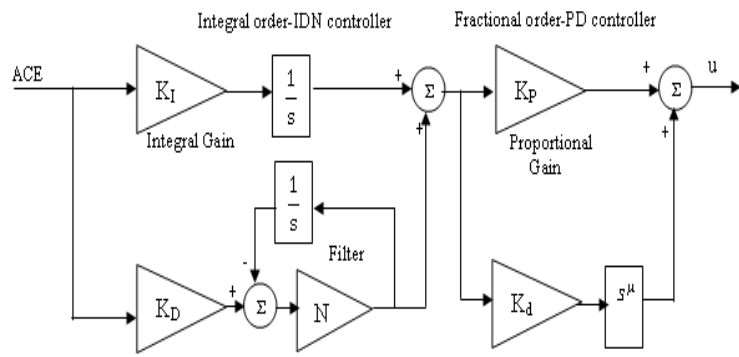

Fig.1 Proposed IDN-FOPD controller

\section{B. Lightning Search Algorithm (LSA)}

It is a trademark wonder dependent meta heuristic calculation depending on the component of lightning that includes proliferation of pioneer step [11]. A bit of atoms of water dense in a thundercloud can split in irregular headings, called as shots. This is viewed as the quick particles termed as shots. The underlying populace calculation size is addressed by the shots and the speed of the shot is showed in (4).

$$
v_{p}=\left[1-\left(\frac{1}{\sqrt{1-\left(\frac{v_{o}}{c}\right)^{2}-\left(\frac{s F_{i}}{m c^{2}}\right)}}\right)^{-2}\right]^{-1 / 2}
$$

where $v_{0}$ is the projectile initial velocity, $\mathrm{m}$ is the projectile mass, $F_{i}$ is the steady ionization rate, c is the light speed and $s$ is the way voyaged length. Subsequently, shot can possibly ionize or research an immense space if mass is less and journey way is high. In this manner, overall vitality of progression head reins the investigation along with misuse of calculation. The shot is forking and improves the horrendous arrangement of populace and the forking point is illuminated to keep the populace size. In the calculation, 3 sorts of shots are acquainted with address the advance pioneer improvement. These are change shots builds up the number of inhabitants in initial step pioneer, space shot which endeavor to accomplish the best location and lead shot addresses the best location among all populace. As the change shots are launch in an irregular course, it very well may be addressed by an arbitrary quantity from uniform likelihood dispersion work, as illustrated in (5). 


$$
f\left(x^{T}\right)=\left\{\begin{array}{lc}
\frac{1}{b-a} ; & a \leq x^{T} \leq b \\
0 ; & x<a, x^{T}>b
\end{array}\right\}
$$

where $\mathrm{x}^{\mathrm{T}}$ is the number generated in random to give the solution. While $\mathrm{i}$ and $\mathrm{b}$ are the boundaries of lower and upper solution space. Following to leader tips of $\mathrm{N}$ step, it projects by ionizing the old leader area utilizing energetic projectiles. The location of the space projectile is calculated by using the probability density function (PDF) as given in equation (6).

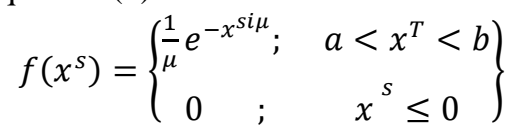

where, $\mu$ is the shaping parameter and $\mu$ iis is the distance between lead and space projectiles. The location of a exacting space projectile is illustrated by (7).

$$
P_{i-n e w}^{s}=P_{i}^{s} \mp \exp \operatorname{rand}_{i}\left(\mu_{i}\right)
$$

On the off chance that the shot vitality isn't more essential than the progression chief, the new location of the space shot doesn't guarantee the proliferation of the ventured pioneer to grow the channel. In the event that it isn't generally, it will advance toward turning into a lead shot. The typical PDF of a lead projectile with scale parameter $\sigma$ is shown in (8).

$$
f\left(x^{L}\right)=\frac{1}{\sigma \sqrt{2 \pi}} e^{-\left(x^{L}-\mu\right)^{2} / 2 \sigma^{2}}
$$

It is expected to obtain a shape parameter for space projectile while the scale parameter decreases exponentially. The lead projectile location is shown in (9).

$$
P_{\text {new }}^{L}=P^{L}+\text { normrand }_{i}\left(\mu_{L}, \sigma_{L}\right)
$$

On off chance that the new location of the lead shot intends a respectable arrangement, at that point the progression head is increased along with the lead shot location is refreshed. Therefore, abuse and investigation are carried out by lead and space shots to locate ideal arrangement. The investigation is addressed by irregular exponential conduct of the space shot and the misuse procedures are constrained by lead shot using arbitrary pursuit. The LSA control parameters are size of the populace, most extreme emphasis and channel time.

\section{CONTROL DESIGN FOR HES AND IPFC UNIT IN AGC LOOP}

The Linearized decrease model test framework with HES and IPFC units for the control configuration has showed up in Fig 2. Where the dynamic of the representative framework is disposed of by fixing the mechanical contributions to be unfaltering since the reaction of senator is much slower than that of HES or IPFC units. It is seen that the IPFC arranged between two regions is successful to balance out the between territory swaying mode just, and afterward the HES is fit for providing the vitality for power framework is to be appropriate for the inactivity mode control. The HES is demonstrated as an active power source to area 1 with gain steady $\mathrm{K}_{\mathrm{HES}}$ and time consistent $\mathrm{T}_{\mathrm{HES}}$. The IPFC is demonstrated as a power flow controller for tie line with time consistent $\mathrm{T}_{\mathrm{IPFC}}$. From Fig 2 , the state equation is derived as follows.

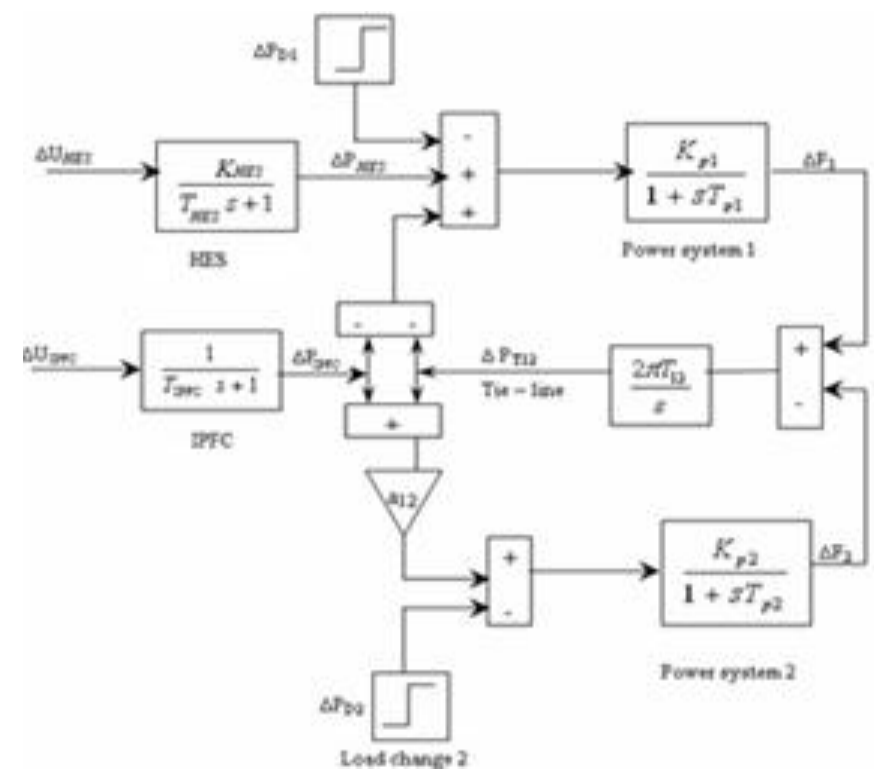

Fig.2 Linearized reduction model for the test system with HES and IPFC unit

$$
\begin{aligned}
& {\left[\begin{array}{c}
\Delta \dot{F}_{1} \\
\Delta \dot{P}_{T 12} \\
\Delta \dot{F}_{2}
\end{array}\right]=\left[\begin{array}{ccc}
-1 / T_{p 1} & -k_{p 1} / T_{p 1} & 0 \\
2 \pi T_{12} & 0 & -2 \pi T_{12} \\
0 & a_{12} k_{p 2} / T_{p 2} & -1 / T_{p 2}
\end{array}\right]\left[\begin{array}{c}
\Delta F_{1} \\
\Delta P_{T 12} \\
\Delta F_{2}
\end{array}\right]+} \\
& {\left[\begin{array}{cc}
k_{p 1} / T_{p 1} & -k_{p 1} / T_{p 1} \\
0 & 0 \\
0 & a_{12} k_{p 2} / T_{p 2}
\end{array}\right]\left[\begin{array}{c}
\Delta P_{H E S} \\
\Delta P_{I P F C}
\end{array}\right]}
\end{aligned}
$$

\section{A. Control design of HES unit}

The structure procedure starts with the decrease of two region framework into one region which addresses the Inertia center strategy for the general framework. The HES controller is structured in proportionate one region framework to decrease the recurrence difference of the latency. The identical framework will be determined using expecting the synchronizing coefficient T12 to be gigantic from the equation of $\Delta P_{T 12}$ in $\mathrm{Eq}$ (10).

$$
\frac{\Delta P_{T 12}^{\cdot}}{2 \pi T_{12}}=\Delta F_{1}-\Delta F_{2}
$$

Let us assume Synchronous coefficient $\left(\mathrm{T}_{12}\right)$ is infinity, then Eq (11) becomes $\Delta \mathrm{F}_{1}=\Delta \mathrm{F}_{2}$. Expanding in Eq (10), $\Delta \dot{F}_{1}$ and $\Delta \dot{F}_{2}$ multiplying by $T_{p 1} / k_{p 1}$ and $T_{p 2} / a_{12} k_{p 2}$ respectively.

$$
\begin{aligned}
& \left(T_{p 1} / k_{p 1}\right) \Delta F_{1}=-\left(1 / k_{p 1}\right) \Delta F_{1}-\Delta P_{T 12}+\Delta P_{H E S}- \\
& \Delta P_{I P F C}
\end{aligned}
$$

$$
\left(T_{p 2} / a_{12} k_{p 2}\right) \dot{\Delta F_{2}}=-\left(1 / a_{12} k_{p 2}\right) \Delta F_{2}+\Delta P_{T 12}+\Delta P_{I P F C}
$$

Sub $\Delta F_{1}=\Delta F_{2}=\Delta F$ and summing Eq (12) and (13) we get

$$
\begin{aligned}
& \Delta \dot{F} \\
& =\left(-1 / k_{p 1}-\left(1 / a_{12} k_{p 2}\right)\right) /\left(\left(T_{p 1} / k_{p 1}\right)+\left(T_{p 2} / a_{12} k_{p 2}\right)\right) \\
& +\left(1 /\left(\left(T_{p 1} / k_{p 1}\right)+\left(T_{p 2} / a_{12} k_{p 2}\right)\right)\right) \Delta P_{H E S}+C \Delta P_{D}
\end{aligned}
$$


The $\Delta \mathrm{P}_{\mathrm{D}}$ which is system load changed is considered and the control function is given below,

$\Delta \mathrm{P}_{\mathrm{HES}}=-\mathrm{K}_{\mathrm{HES}} \Delta \mathrm{F}$ is applied then.

$\Delta F=\frac{C}{s+A+K_{H E S} B} \Delta P_{D}$

Where

$A=$

$$
\begin{gathered}
\left(-1 / k_{p 1}-\left(1 / a_{12} k_{p 2}\right)\right) /\left(\left(T_{p 1} / k_{p 1}\right)+\left(T_{p 2} / a_{12} k_{p 2}\right)\right) \\
B=1 /\left(\left(T_{p 1} / k_{p 1}\right)+\left(T_{p 2} / a_{12} k_{p 2}\right)\right)
\end{gathered}
$$

In (15) the final values with $\mathrm{K}_{\mathrm{HES}}$ equals to zerz and $\mathrm{K}_{\mathrm{HES}}$ non equals to zero are $\mathrm{C} / \mathrm{A}$ and $\mathrm{C} /\left(\mathrm{A}+\mathrm{K}_{\mathrm{HES}} \mathrm{B}\right)$. The percent reduction is given in (16).

$\left(C / A+K_{H E S} B\right) /(C / A)=R / 100$

The control gain of HES unit is expressed as

$K_{H E S}=(A / B R) *(100-R)$

\section{B. IPFC Controller design}

It is intended to improve the damping of the between territory so as to remove the between territory mode from the framework. Equation (10), covering deteriorations is applied. At that point, one subsystem which jam the between region mode is addressed by,

$$
\begin{gathered}
{\left[\begin{array}{c}
\Delta \dot{F}_{1} \\
\Delta P_{T 12}
\end{array}\right]=\left[\begin{array}{cc}
-1 / T_{p 1} & -k_{p 1} / T_{p 1} \\
2 \pi T_{12} & 0
\end{array}\right]\left[\begin{array}{c}
\Delta F_{1} \\
\Delta P_{T 12}
\end{array}\right]+} \\
{\left[\begin{array}{c}
-k_{p 1} / T_{p 1} \\
0
\end{array}\right]\left[\Delta P_{I P F C}\right]}
\end{gathered}
$$

The control inspiration driving the interline power flow controller is to drench the pinnacle estimation of recurrence change in zone 1 after an unexpected changes at the heap request. As the framework in (18) is the second request wavering framework, the overshoot rate Mp (new) would be indicated for the control structure. Mp (new) is illustrated as a component of the damping proportion as,

$$
M_{P \text { new }}=e^{\left(-\pi \delta / \sqrt{1-\delta^{2}}\right)}
$$

The genuine and imaginary pieces of eigen value after the control are given as $\alpha_{s}=\delta \omega_{n}$ and $\beta_{s}=\omega_{n \sqrt{1-\delta^{2}}}$. Where $\mathrm{w}_{\mathrm{n}}$ is the undamped natural frequency and the eigenvalue technique derive to the feedback conspire as,

$$
\Delta P_{I P F C}=-k_{1} \Delta F_{1}-k_{2} \Delta P_{T 12}
$$

\section{MODELLING AND DESIGN OF 2 AREA WIND-SOLAR-THERMAL SYSTEM IN DEREGULATED POWER FRAMEWORK}

In the deregulated framework, Discos zone can bond with Gencos. A Disco has the opportunity in having simultaneousness to any Genco towards comprehension of intensity. Such trades are called two-sided trades [3]. The entirety of the trades must be cleared through a reasonable part called an ISO. In this two-region, the breeze sun oriented warm power framework with HES and IPFC units is considered in which every domain has 2 Gencos and 2 Discos has appeared in Fig 3. The AGC execution is recognized on the parameters of steam turbine dynamic model. The parameters are believed to be reliant on the age timetables of warm power plants [14]. The dynamic models of the warm bike blend buildup turbine appear in Fig 4. The guideline prerequisites are the time constants TSC, TRH and TCO of Steam Chest (SC), Re-heater (RH) and Cross Over (CO) pipe separately and the power divisions $\mathrm{F}_{\mathrm{HP}}, \mathrm{F}_{\mathrm{IP}}$ and $\mathrm{F}_{\mathrm{LP}}$ of High, Intermediate and Low Pressure turbines independently. The customary estimations at different time constants and power bits of thermal reheat turbine can be intended for various generation plans by expelling the warmth balance information is showed up in the appendix [14]. Discos can have agreement control from any Gencos and ISO needs to administer these agreements. DPM is a lattice where the amount of lines is comparable to the amount of Gencos along with the amount of segments is proportionate to the amount of Discos in the framework Every entry in this grid can be considered for the bit of a overall burden shrunk by a Disco through a Genco. The aggregate of significant number of sections in a fragment DPM is solidarity. From Fig 3, Let Genco-1, Genco-2, Disco-1, Disco-2 be in region 1 while Genco-3, Genco-4, Disco-3, Disco-4 be in territory number 2 . 


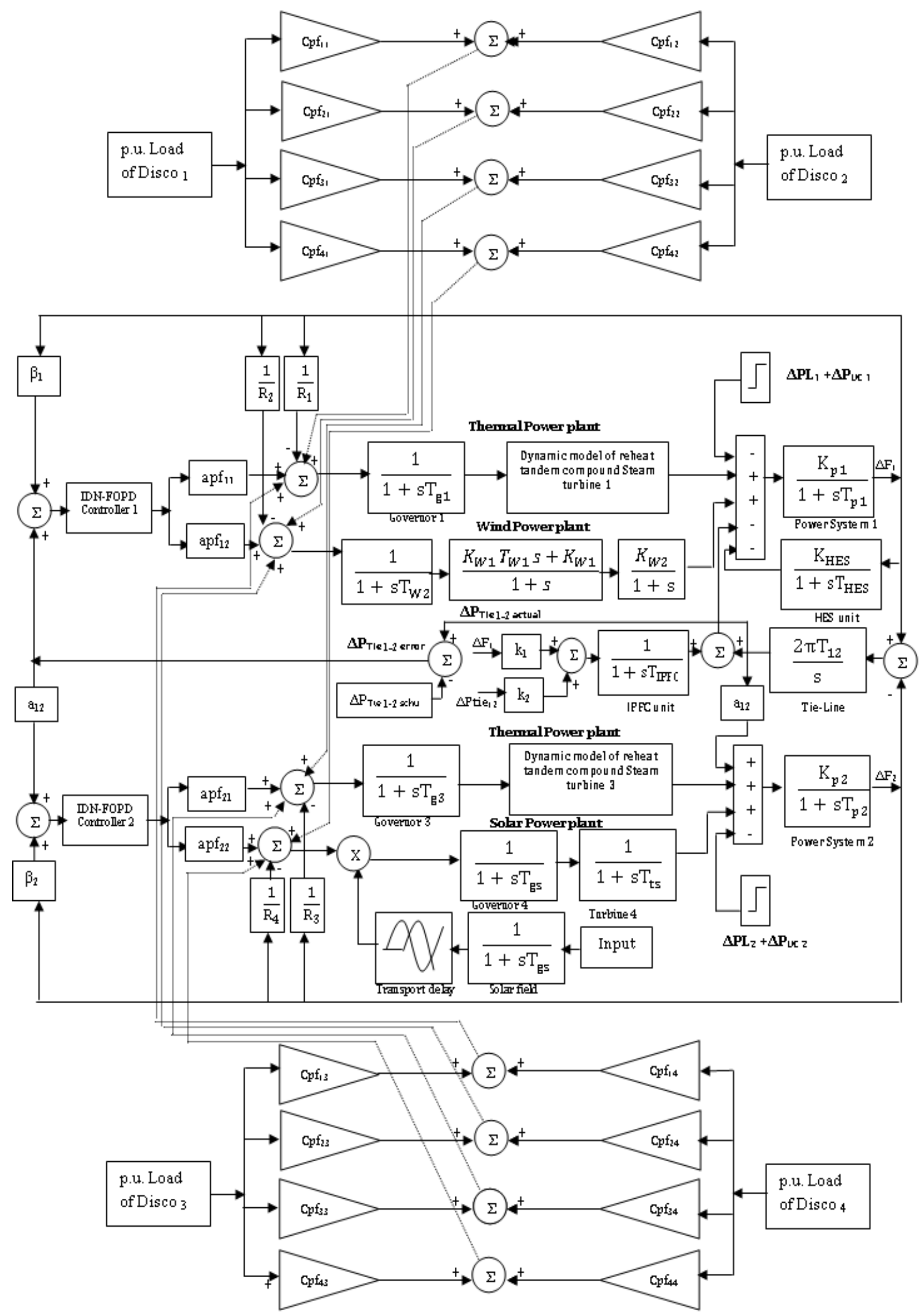

Fig.3 Model of 2 area wind-solar-thermal system with HES and IPFC unit in deregulated power framework (Transfer function model)

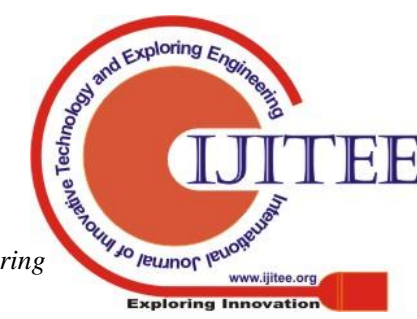




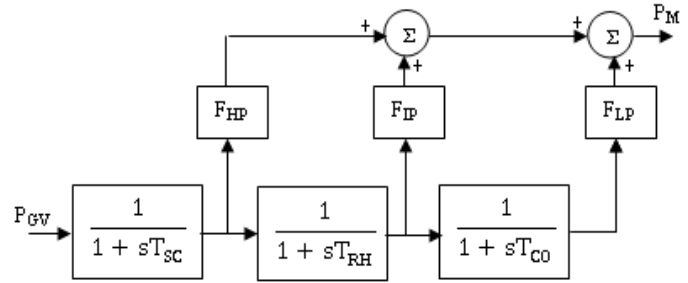

Fig.4 Dynamic model for a reheat tandem compound steam turbine

$$
D P M=\left[\begin{array}{llll}
c p f_{11} & c p f_{12} & c p f_{13} & c p f_{14} \\
c p f_{21} & c p f_{22} & c p f_{23} & c p f_{24} \\
c p f_{31} & c p f_{32} & c p f_{33} & c p f_{34} \\
c p f_{41} & c p f_{42} & c p f_{43} & c p f_{44}
\end{array}\right]
$$

where $c p f$ is the contract participation factor. Per unit MW load of a Disco. The steady state power flow on the tie line is expressed as,

$$
\begin{aligned}
& \Delta P_{\text {Tie 12 } 12}^{\text {scheduled }}= \\
& \sum_{i=1}^{2} \sum_{j=3}^{4} c p f_{i j} \Delta P_{L j}-\sum_{i=3}^{4} \sum_{j=1}^{2} c p f_{i j} \Delta P_{L j}
\end{aligned}
$$

The normal tie line power is illustrated as (23) and its power error in (24).,

$$
\begin{aligned}
& \Delta P_{\text {Tie } 12}^{\text {actual }}=\frac{2 \pi T_{12}}{s}\left(\Delta F_{1}-\Delta F_{2}\right) \\
& \Delta P_{\text {Tie 12 }}^{\text {Error }}=\Delta P_{\text {Tie } 12}^{\text {actual }}-\Delta P_{\text {Tie 12 }}^{\text {scheded }} \\
& A C E_{1}=\beta_{1} \Delta F_{1}+\Delta P_{\text {Tie12 }}^{\text {Error }} \\
& A C E_{2}=\beta_{2} \Delta F_{2}+a_{12} \Delta P_{\text {Tie12 }}^{\text {Error }}
\end{aligned}
$$

The desired absolute power generation of Genco at ith location as far as DPM entries can be determined as,

$$
\Delta P_{m i}=\sum_{j=1}^{4} c p f_{i j} \Delta P_{L j}
$$

Coefficients that circle ACE to Gencos are named as ACE Participation Factors (apfs)..

\section{SIMULATION RESULTS AND OBSERVATIONS}

In this assessment two-region wind-sunlight based warm deregulated power framework is meticulous for the examination with various kind's exchanges. Every zone has 2 Gencos units and two discos units. The model of the structure under investigation was made in the MATLAB/Simulink condition. The apparent parameters are given in the Appendix. Lightning Search Algorithm (LSA) method is considered for perfect tuning IDN-FOPD controller for the AGC hover of a two-zone wind-sun based warm deregulated power framework with warm bike blend buildup turbine. The perfect arrangement of control information sources is taken for development issue and the target work in Eqn (1) is resolved to use the recurrence control deviation regions and power changes in tie line. The ideal IDN-FOPD gain estimations of the test framework are resolved LSA system. These IDN-FOPD controllers are actualized in a proposed test framework for various sorts of exchanges and differentiated and PI and FOPID controllers.

\section{Scenario 1: Poolco based transaction}

Gencos participate just in the load following control of their areas. It is assumed that a huge step load of 0.2 p.u MW is demanded by each Disco in area 1. Assume that a case of Poolco based contracts among Dicos and available Gencos are simulated with reference to the Disco
Participation Matrix (DPM) shown below referring to (21) is taken as,

$$
D P M_{1}=\left[\begin{array}{llll}
0.5 & 0.5 & 0.0 & 0.0 \\
0.5 & 0.5 & 0.0 & 0.0 \\
0.0 & 0.0 & 0.0 & 0.0 \\
0.0 & 0.0 & 0.0 & 0.0
\end{array}\right]
$$

Disco $_{1}$ and Disco $_{2}$ stipulate identically from their local Gencos, viz., Genco ${ }_{1}$ and Genco $_{2}$. Therefore, $\mathrm{cpf}_{11}=\mathrm{cpf}_{12}=$ 0.5 and $\mathrm{cpf}_{21}=\mathrm{cpf}_{22}=0.5$. In this test system consists of two Gencos and two Discos in each area. The wind and thermal units are Gencos in area-1 and solar and thermal units as Gencos in area-2. The controller is tuned utilizing the LSA method and actualized a two-region interconnected breeze sun oriented warm deregulated power framework for various exchanges. In Fig 5 shows similar transient exhibitions of wind-sunlight based warm power framework for given burden bother. From Fig 5, it tends to be seen that the motions in the territory frequencies and tie-line control deviations have diminished to an impressive degree with LSA tuned IDN-FOPD controller when appeared differently in relation to the yield reactions acquired utilizing PI and FOPID controller. Along these lines, IDN-FOPD can be used as a reasonable helper controller in both the AGC circle and the examination of the succeeding sub-segments is proceeded by thinking about a comparative controller.Scenario 2: Bilateral based transaction

Here all the Discos have contract with the Gencos and the following Disco Participation Matrix (DPM) referring to Eq (21) is considered as

$$
D P M_{2}=\left[\begin{array}{cccc}
0.2 & 0.3 & 0.1 & 0.4 \\
0.3 & 0.2 & 0.3 & 0.1 \\
0.25 & 0.1 & 0.25 & 0.3 \\
0.25 & 0.4 & 0.35 & 0.2
\end{array}\right]
$$

In this case, the Disco $_{1}$, Disco $_{2}, \operatorname{Disco}_{3}$ and Disco $_{4}$, demands of $0.1 \mathrm{pu} . \mathrm{MW}$ for each from Gencos as defined by cpf in the $\mathrm{DPM}_{2}$ matrix and each Gencos participates in AGC as defined by the following ACE participation factor $\operatorname{apf}_{11}=\operatorname{apf}_{12}=0.5$ and $\operatorname{apf}_{21}=\operatorname{apf}_{22}=0.5$. The active power model of IPFC unit is fitted in the tie-line near area1 and HES unit is installed in areal to examine its effect on the power system performance. The gain values of HES unit is $\mathrm{K}_{\mathrm{HES}}=0.902$ using Eqn (17) for the given value of speed regulation coefficient $(\mathrm{R})$. The purpose of incorporating IPFC unit is to damp out the peak value of frequency deviations in both area and tie-line power deviations. Since the system Eqn (18) is second order oscillations system, the feedback gains $\mathrm{k}_{1}$ and $\mathrm{k}_{2}$ are found using Eqn (20) for specified peak over shoot $M p$ (new). The feedback gain values IPFC is $\mathrm{k}_{1}=-0.645$ and $\mathrm{k}_{2}=-1.08$ for $M p(n e w)=2 \%$. The optimum IDN-FOPD controller gain estimations of the test system with HES and IPFC units for various case studies are determined LSA technique. The comparative transient performances of two-area wind-solar-thermal deregulated power system with HES and IPFC units using FOPID controller for the bilateral transactions are appeared in Fig 6. It might be reasoned that the proposed design idea

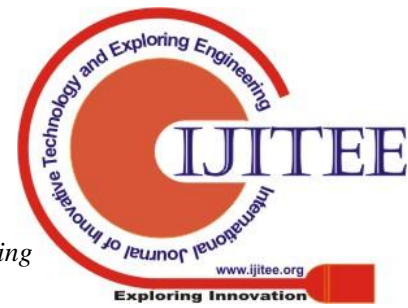


effectively damps out the inertia mode and inter-area mode on account of the coordinated control action of HES and IPFC units and is found to be more effective to reduce the deviations in frequency of the 2 area system

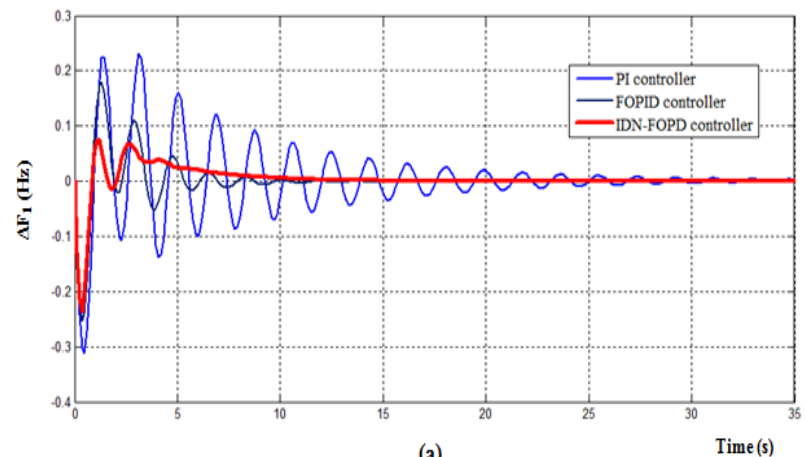

(a)

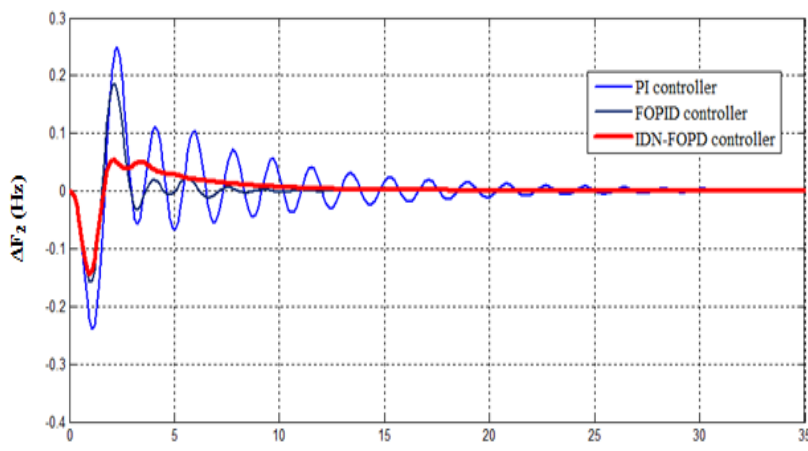

(b)

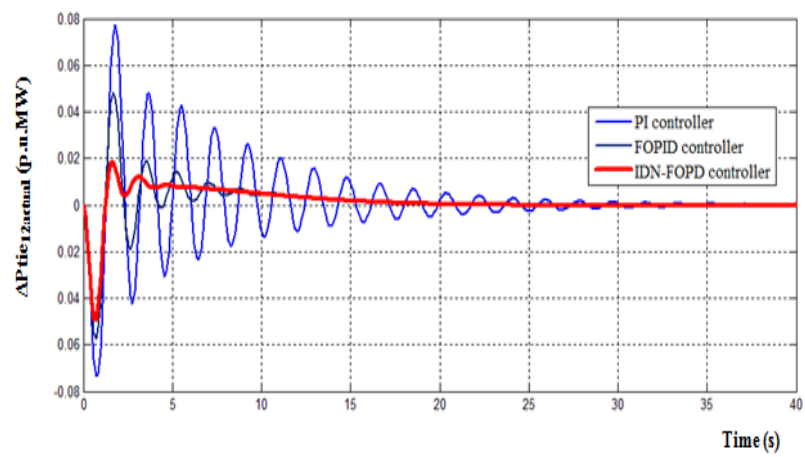

(c)

Fig .5 Dynamic responses (a) frequency deviations of area 1 , (b) frequency deviations of area 2 , (c) tie-line power changes for a 2 area wind-solar-thermal system utilizing different controller types (Poolco based transaction)

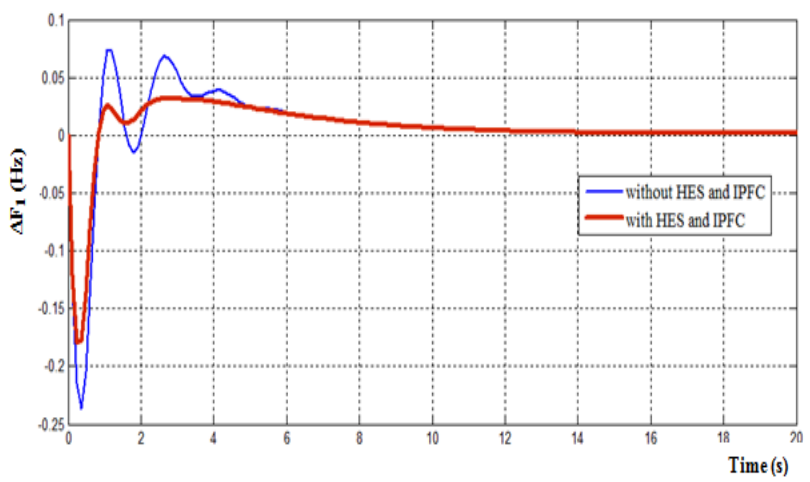

(a)

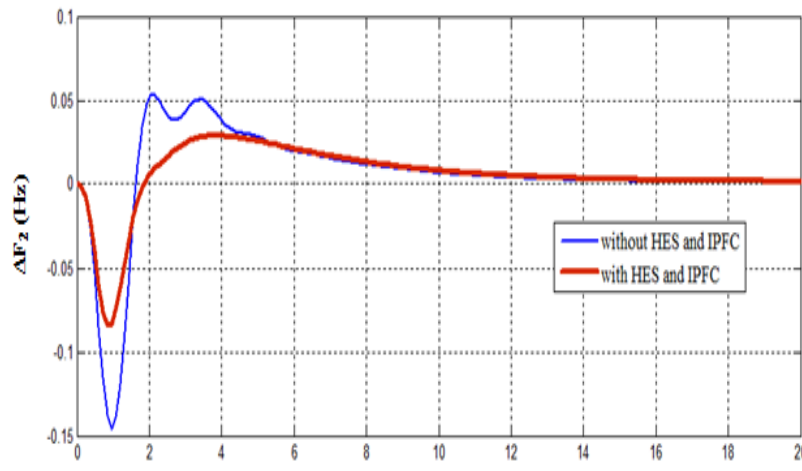

(b)

Time(s)

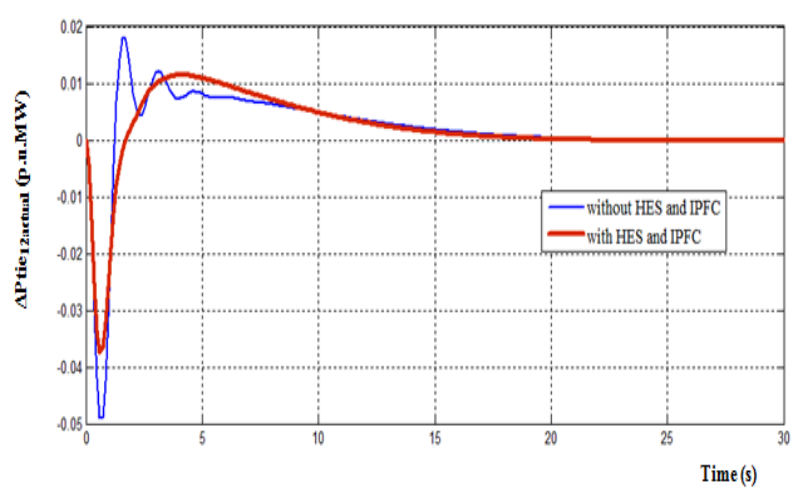

(c)

Fig.6 Dynamic responses (a) frequency deviations of area1, (b) frequency deviations of area number 2, (c) power deviations of tie line for a two area wind-solar-thermal system using IDN-FOPD controller without and with HES and IPFC units (Bilateral based transaction)

\section{CONCLUSION}

The IDN-FOPD controllers are planned utilizing the LSA strategy and actualized in two territory wind-sun powered warm interconnected power frameworks without and with HES and IPFC units for various types of exchanges. The different mimicked results exhibit that the LSA based IDNFOPD performs better than some old style controllers with respect to diminished pinnacle deviations just as settling time of recurrence deviation in the two territories and tieline control motions in AGC circle. In these examinations evidently reveal that the IDN-FOPD controller is incredibly improved than Integral request and fragmentary request controllers in upgrading framework control exhibitions. The planned utilization of HES and IPFC units in the AGC circle is furthermore compelling in settling recurrence unsettling influences during two-sided exchanges by the organizations. Subsequently, the coordination of HES-IPFC units is adequately applied for balancing out the heap recurrence issues in a two-zone wind-sun based warm framework under a deregulated situation. 


\section{APPENDIX}

\section{Control area and Gencos parameters and parameter pertaining to HES and IPFC unit [12, 13 and 14]}

\begin{tabular}{|l|l|l|}
\hline \multicolumn{1}{|c|}{ Parameters } & Area1 & Area 2 \\
\hline Area capacities & $1000 \mathrm{MW}$ & $1000 \mathrm{MW}$ \\
\hline $\begin{array}{l}\text { Rating of single } \\
\text { generating } \\
\text { machine }\end{array}$ & $500 \mathrm{MW}$ & $500 \mathrm{MW}$ \\
\hline Kp (Hz/p.u.MW) & 120 & 120 \\
\hline Tp (sec) & 20 & 20 \\
\hline B (per unit MW / Hz) & 0.425 & 0.425 \\
\hline R (Hz / per unit MW) & $\mathrm{R}_{1}$ and & $\mathrm{R}_{3}$ \\
$\mathrm{R}_{4}=2.4$
\end{tabular}

\section{REFERENCES}

1 Kundur, Prabha, Neal J. Balu, and Mark G. Lauby. Power system stability and control. Vol. 7. New York: McGraw-hill, 1994.

2 Elgerd, O.I. Electric energy systems theory: an introduction. Tata McGraw Hill Education Pvt. Ltd, New Delhi, India, 2014.

3 Donde, Vaibhav, M. A. Pai, and Ian A. Hiskens. "Simulation and optimization in an AGC system after deregulation." IEEE transactions on power systems, Vol.16, No.3, pp. 481-489, 2001.

4 Pardeep Nain, K.P.Singh Parmar and A.K. Singh, "Automatic Generation Control of an Interconnected Power System Before and After Deregulation", International Journal of Computer Applications, Vol. 61, No.15, pp.11-16, 2013.

5 Saha, Debdeep, and L. C. Saikia. "Performance of FACTS and energy storage devices in a multi area windhydro-thermal system employed with SFS optimized I-
PDF controller." Journal of Renewable and Sustainable Energy, Vol.9, No. 2, pp. 024103, 2017.

6 Gnanavel $\mathrm{C}$ and Albert Alexander S, "Experimental Validation of an Eleven Level Symmetrical Inverter Using Genetic Algorithm and Queen Bee Assisted Genetic Algorithm for Solar Photovoltaic Applications", Journal of Circuits, Systems and Computers, Vol.27. No.13, pp. (185021)1-23, 2018.

7 Baskar, B., and B. Paramasivam, "FOPID controller based AGC loop of a two-area Solar-Thermal deregulated Power System with Vanadium Redox Flow Battery", International Journal for Research in Engineering Application \& Management, Vol-04, Issue12, pp.245-253, 2019.

8 Saikia, Lalit Chandra, and Shashi Kant Sahu, "Automatic generation control of a combined cycle gas turbine plant with classical controllers using firefly algorithm", International Journal of Electrical Power \& Energy Systems, Vo. 53, pp. 27-33, 2013.

9 Sahu RK, Panda S, Rout UK, "DE optimized parallel 2DOF PID controller for load frequency control of power system with governor dead-band nonlinearity", International Journal of Electrical Power \& Energy Systems Vol.49, pp.19-33, 2013.

10 Javad M, Kazem Z, Mehrdad TH. "Applying fractional order PID to design TCSC-based damping controller in coordination with automatic generation control of interconnected multi-source power system", Engineering Science and Technology, an International Journal, Vol.20, No.1, pp.1-17, 2017.

11 Rajbongshi, R and Saikia, L.C "Combined control of voltage and frequency of multi-area multisource system incorporating solar thermal power plant using LSA optimised classical controllers", IET Generation, Transmission \& Distribution, Vol.11, No.10, pp.24892498, 2017

12 Shankar, R., Chatterjee, K. and Bhushan, R., "Impact of energy storage system on load frequency control for diverse sources of interconnected power system in deregulated power environment", International Journal of Electrical Power and Energy Systems, Volume 79, pp.11-26, 2016

13 Chidambaram, I.A. and Paramasivam, B., "Optimized load-frequency simulation in restructured power system with redox flow batteries and interline power flow controller", International Journal of Electrical Power \& Energy Systems, Vol.50, pp.9-24, 2013.

14 Nikhil Pathak, Ashu Verma, and Terlochan Singh Bhatti, "Automatic generation control of thermal power system under varying steam turbine dynamic model parameters based on generation schedules of the plants", The Journal of Engineering, Vol.24, pp.1-13, 2

15 Albert Alexander S \& Manigandan T, "Reduction of Voltage Harmonics in Solar Photovoltaic fed Inverter of Single phase Stand Alone Power System" Journal of Solar Energy Engineering - Transactions of the ASME, Vol.136, No.4, pp.044501 (1)-(4), November 2014.

\section{AUTHORS PROFILE}

Sh V Suresh Babu is pursuing Ph.D since 2015 in the Department of Electrical \& Electronics Engineering under Visvesvaraya Technological University, Belgaum, Karnataka, India. He is currently working as Assistant Director at NPTI (PSTI), Ministry of Power, Government of India, Bangalore. His area of research is Renewable Energy Sources and 
Grid Integration. He has been involving as a "Power System Operation \& Control" trainer for Load Despatch Engineers across the country, since 2011. His area of expertise also includes Distributed Generation, Smart Grids, System Operation, Protection, Testing and Energy Efficiency of Power Transmission and Distribution Systems.

Dr K Shanmukha Sundar has obtained Ph.D in Electrical \& Electronics Engineering Sciences during the year 2010 from Visvesvaraya Technological University, Belgaum, Karnataka, India. He is presently working as Professor, Department of Electrical and Electronics Engineering, Dayananda Sagar Academy of Technology and Management, Bangalore. His area of research includes FACTS Controllers, Power System Operations \& Controls, Voltage Stability, Renewable Energy Sources, Control Systems and Optimization of Power Systems, Power Electronics and Drives. 